\title{
Un tutor inteligente, afectivo y configurable para el aprendizaje de números naturales de 3er grado
}

\author{
María Lucia. Barrón-Estrada, Ramón Zatarain-Cabada, Margarita Aranda-Ortega, \\ H.E. Gómez-Pérez, José Mejía-Arredondo \\ Instituto Tecnológico de Culiacán, Juan de Dios Bátiz 310 pte., Col. Guadalupe, \\ Culiacán Sinaloa, CP 80220, México \\ \{rzatarain, lbarron, maranda, hgomez, jmejia\}@itculiacan.edu.mx
}

\begin{abstract}
Resumen. Este articulo presenta el diseño e implementación de un Sistema Tutor Inteligente (STI) capaz de ser configurado por diferentes expertos en áreas consideradas importantes para un STI. Se incorporan las características necesarias para que sean los expertos los que provean las herramientas necesarias para dotar al STI con la capacidad que requiere, para lograr un mayor impacto en el aprendizaje de los números naturales de los niños de 3er grado de primaria. Al STI le pueden ser modificados los conjuntos y reglas difusas, el componente de reconocimiento de afecto, el examen diagnóstico y el reconocimiento de estilo de aprendizaje. Esto le permite al STI cambiar conforme requiera el entorno.
\end{abstract}

Palabras clave: computación afectiva, sistemas tutores inteligentes, redes neuronales, sistemas difusos, configuración externa.

\section{Introducción}

La utilidad del estudio de las emociones en diferentes áreas se ha visto incrementada debido al impacto que estas tienen en el desempeño de nuestra actividades [6,7]. En los últimos años los STI han incorporado la habilidad para reconocer el estado afectivo de los estudiantes y, con ello, ser capaces de cambiar la manera de interactuar a partir de esta entrada [1-5]. La detección de emociones en los usuarios de los STI en muchos trabajos se realiza utilizando sensores especiales como sillas de postura o brazaletes conductivos [8]. Los cuales resultan intrusivos y molestos para los usuarios. Esto ya ha sido atacado anteriormente mediante aproximaciones que incluyen la detección de emociones dentro del tutor [9].

En el presente trabajo se busca integrar dentro del tutor diferentes aspectos como son el estado emocional y el estilo de aprendizaje del estudiante, además de su nivel de conocimiento sobre los números naturales. Esto se logra mediante el uso de una red de Kohonen en un servidor dedicado fuera del entorno del STI para el reconocimiento de 
emociones, se manejan reglas y conjuntos difusos para detectar el nivel del estudiante en base a la resolución de los ejercicios y un examen especializado para el reconocimiento del estilo de aprendizaje [12].

Un STI es tan inteligente como el conocimiento introducido por sus expertos, por lo que al implementar una plataforma que sea capaz de ser utilizada por múltiples especialistas, obtendrá experiencia que le permitirá igualar e incluso superar a cualquier otro STI. Es por ello que mostramos resultados haciendo comparación con diversos tutores [15-21] que son considerados los mejores en sus respectivas áreas.

El artículo está dividido de la siguiente manera: la sección 2, Configuración del STI explica como la capacidad de configuración por distintos expertos, aporta una característica fundamental para la personalización de los entornos en que pueda ser utilizado el STI. La sección 3, Dominio del STI describe el subconjunto de lecciones que se utilizaron del libro de matemáticas de tercer grado de primaria [23]. La sección 4, Arquitectura del STI muestra la estructura y relación de los componentes más importantes del tutor, haciendo énfasis en los componentes que manejan la parte de inteligencia artificial. La sección 5, Pruebas y Evaluación del STI considera diversos aspectos que fueron seleccionados para realizar la comparación con múltiples STI. Por último las Conclusiones expresan los resultados obtenidos.

\section{Configuración del STI}

Una característica que distingue al STI es su capacidad para que un experto pueda configurar cualquier tema de un dominio en específico. En el caso de las pruebas de diagnóstico o ejercicios, el maestro es capaz de introducir y cambiar las lecciones que el considere son las mejores para sus estudiantes.

Lo mismo aplica para el componente neurodifuso, un experto computacional puede acceder a una API para ingresar o modificar las reglas y conjuntos difusos, de tal manera que cuando un estudiante cometa un error o genere un acierto, esta acción refleje la clasificación que tiene el STI sobre el conocimiento actual de este.

De la misma forma, el examen de estilo de aprendizaje puede ser cambiado por un psicólogo u otro profesional competente, para determinar de qué manera aprende mejor el estudiante y que el STI obtenga mayor información para personalizar la enseñanza con el fin de mejorar el conocimiento del estudiante.

El STI utiliza un componente afectivo para procesar imágenes recolectadas mientras que el estudiante se encuentra respondiendo ejercicios. Esta API extensible fue concebida con el propósito de proveer al STI con una herramienta para cambiar la manera en la que trabaja el reconocimiento de afecto.

Esta capacidad de configuración es implementada por el formato JSON, el cual es avalado internacionalmente como estándar en la transferencia de información web. La 
estructura que debe llevar, puede ser generada por muchas herramientas en línea, facilitándole al experto su uso.

\section{Dominio del STI}

El libro de texto gratuito de la SEP, Matemáticas 3er. Grado, contiene 17 temas, como se muestra en la Fig. 1, los cuales se abordan en diferentes lecciones a lo largo del libro.

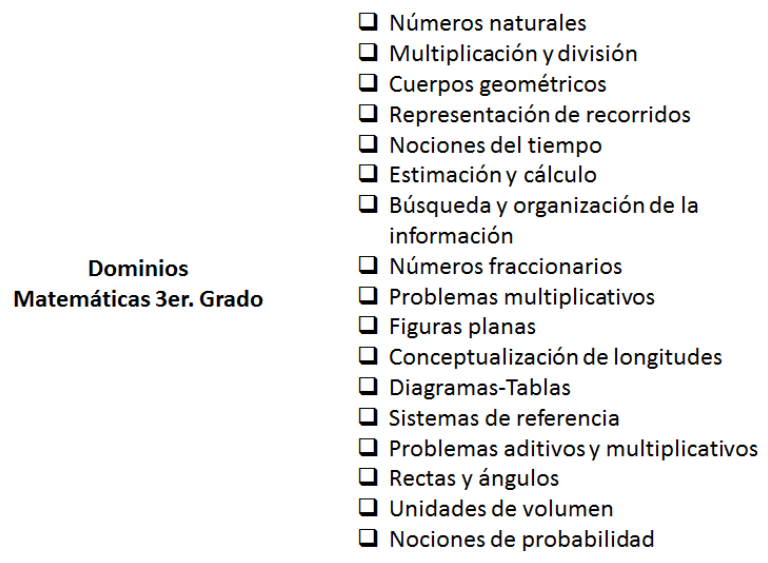

Fig. 1. Dominios dentro del Libro de Texto Gratuito de la SEP. Matemáticas 3er. Grado.

El libro se encuentra organizado con una estructura jerárquica que cuenta con lo siguiente:

$\checkmark$ Bloques. Contiene cinco bloques de conocimiento, organizados secuencialmente como Bloque 1 ... Bloque 5 .

$\checkmark$ Aprendizajes esperados. Cada bloque establece una serie de objetivos

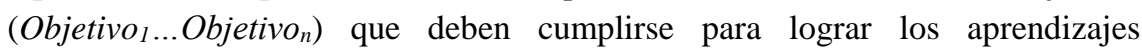
esperados.

$\checkmark \quad$ Lecciones. Cada lección define un apartado llamado Lo que conoce el estudiante que es un diagnóstico previo a cada lección y una serie de actividades (Actividad $_{1} \ldots$ Actividad $_{n}$ ), que describen lo que el estudiante debe realizar. Las actividades a su vez, sugieren otras acciones como son: Consultas a otras fuentes, las Actividades reto y Datos interesantes relacionadas con la actividad. 
$\checkmark$ Integración de lo aprendido. Se presenta una serie de problemas $\left(\right.$ Problema $_{1} \ldots$ Problema $\left._{n}\right)$, relacionados con la lección de estudio para que el estudiante pueda aplicar su aprendizaje resolviendo problemas.

$\checkmark$ Evaluación. Contiene una serie de ejercicios correspondientes a la lección presentada, para evaluar con ellos, sí el estudiante ha alcanzado los objetivos.

$\checkmark$ Autoevaluación. Esta sección se incluye para que el estudiante pueda evaluarse a si mismo de forma Procedural y Actitudinal al final de cada lección.

Para el diseño del Sistema Tutor Inteligente (STI) se definió el dominio de los Números Naturales. En el libro de Matemáticas de tercer grado, este tema se encuentra distribuido en varios Bloques, que contienen diferentes lecciones que definen los objetivos que el estudiante debe cumplir al momento de la evaluación procedural. En la

Tabla 1, se presentan los bloques, las lecciones, los objetivos y las habilidades por adquirir correspondientes al dominio de los Números Naturales.

Tabla 1. Objetivos dentro del dominio de los números naturales.

\begin{tabular}{|c|c|c|c|}
\hline Bloque & Lección & Objetivos a cumplir & $\begin{array}{l}\text { Habilidades por adquirir del } \\
\text { estudiante }\end{array}$ \\
\hline 1 & 1 & $\begin{array}{l}\text { Compara y ordena números de cuatro } \\
\text { cifras. }\end{array}$ & $\begin{array}{l}\text { Aprende a agrupar los elementos de } \\
\text { una colección en unidades, decenas, } \\
\text { centenas y unidades de millar. }\end{array}$ \\
\hline 1 & 2 & $\begin{array}{l}\text { Compara y ordena números de cuatro } \\
\text { cifras. }\end{array}$ & $\begin{array}{l}\text { Cuenta los elementos de una } \\
\text { colección para compararlos con otra. }\end{array}$ \\
\hline 1 & 3 & $\begin{array}{l}\text { Utiliza el cálculo mental al restar } \\
\text { dígitos y múltiplos de } 10 \text { menos un dígito. }\end{array}$ & $\begin{array}{l}\text { Identifica regularidades en la } \\
\text { sucesión numérica. }\end{array}$ \\
\hline 1 & 4 & $\begin{array}{l}\text { Obtiene de manera rápida los } \\
\text { productos de dígitos para resolver } \\
\text { problemas u operaciones. }\end{array}$ & $\begin{array}{l}\text { Desarrolla la habilidad de realizar } \\
\text { mentalmente restas con números del } 1 \text { al } \\
\text { 10. }\end{array}$ \\
\hline 2 & 14 & $\begin{array}{l}\text { Identifica y Compara números escritos } \\
\text { como expresiones aditivas y } \\
\text { multiplicativas. }\end{array}$ & 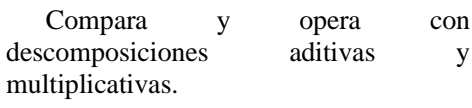 \\
\hline 2 & 15 & $\begin{array}{l}\text { Identifica y Compara números escritos } \\
\text { como expresiones aditivas y } \\
\text { multiplicativas. }\end{array}$ & $\begin{array}{l}\text { Relaciona escrituras aritméticas y } \\
\text { nombres de números. }\end{array}$ \\
\hline 2 & 18 & $\begin{array}{l}\text { Utiliza caminos cortos pata multiplicar } \\
\text { dígitos por } 10 \text {, por } 100 \text { y por sus múltiplos } \\
(20,30.200,300 \text {, etc. })\end{array}$ & $\begin{array}{l}\text { Utiliza una forma práctica para } \\
\text { multiplicar un número por } 10 \text {, por } 20 \text {, por } \\
\text { 30, por } 100 \text {, por } 200 \text {. }\end{array}$ \\
\hline 3 & 27 & $\begin{array}{l}\text { Utiliza el algoritmo para multiplicar } \\
\text { números de tres cifras por un dígito. }\end{array}$ & $\begin{array}{l}\text { Estima el resultado de un cálculo de } \\
\text { suma o resta. }\end{array}$ \\
\hline 3 & 28 & $\begin{array}{l}\text { Resuelve problemas que impliquen } \\
\text { dividir mediante diverso procedimientos. }\end{array}$ & $\begin{array}{l}\text { Identifica el recurso más adecuado } \\
\text { para realizar un cálculo: calculadora, } \\
\text { cálculo mental, cálculo escrito. }\end{array}$ \\
\hline 5 & 47 & $\begin{array}{l}\text { Anticipa lo que pasa con el resultado } \\
\text { de la división cuando el dividendo o el } \\
\text { divisor aumentan o disminuyen. }\end{array}$ & $\begin{array}{l}\text { Utiliza el repertorio multiplicativo } \\
\text { para resolver divisiones. }\end{array}$ \\
\hline
\end{tabular}




\section{Arquitectura del sistema tutor inteligente}

El sistema tutor inteligente cuenta con una arquitectura de capas relajadas que se puede visualizar en la fFig. 2.

\subsection{Capa de visualizador web}

Es utilizada para la presentación del tutor y es con lo que interactúa el usuario. Esta cuenta con dos componentes: Interface: es la interfaz del tutor y está hecho con HTML5, CSS3 y JAVASCRIPT, Login: Provee la comunicación para ingresar al tutor por medio de Facebook actualmente, pero se planea acrecentarlo a google+ y twitter.

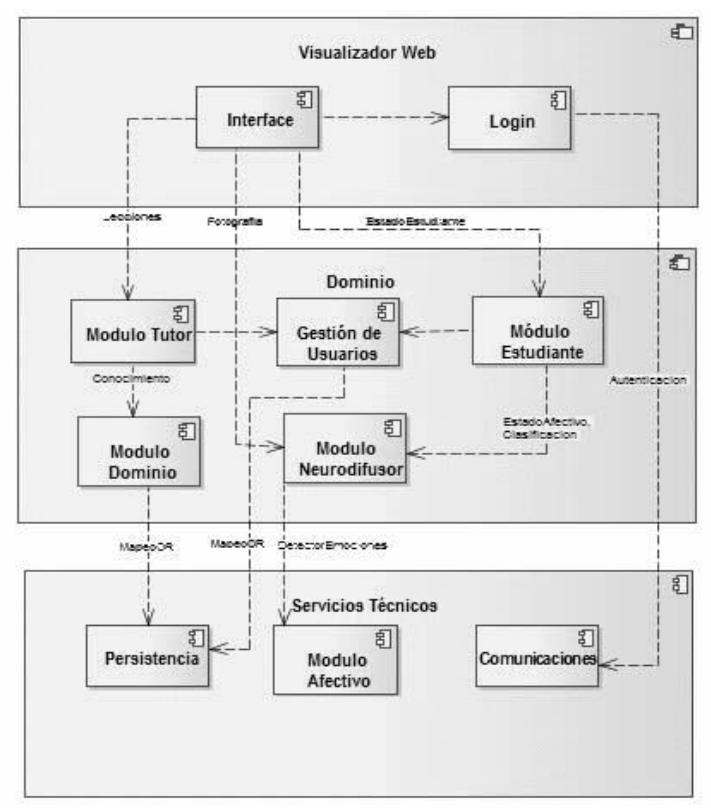

Fig. 2. Arquitectura del sistema tutor inteligente. 


\subsection{Capa de dominio}

La capa de dominio implementa todo lo relacionado con la lógica del tutor, inteligencia artificial y pase de la información hacia el almacenamiento, todo en esta capa está implementado en PHP y consta de cinco componentes.

\subsubsection{Módulo tutor}

Codifica los métodos de enseñanza que sean adecuados para el área de estudio y el alumno. Basado en los datos de las debilidades y fortalezas, nivel de conocimiento, y estilo de aprendizaje, el instructor selecciona el modelo de intervención de enseñanza más apropiado. Por ejemplo, si un estudiante ha sido evaluado como principiante en un determinado ejercicio, el módulo muestra algunos ejemplos paso a paso del procedimiento antes de invitar al usuario a realizarlo el mismo. También puede proporcionar retroalimentación, explicaciones y el entrenamiento a medida que el participante realiza el proceso. Conforme el estudiante gana experiencia, el módulo puede decidir presentar los escenarios cada vez más complejos. También puede decidir tomar el asiento de atrás y dejar que la persona explore libremente los ejercicios, intervenir con las explicaciones y el entrenamiento sólo bajo petición. Además, el módulo se encarga de poder elegir temas y ejemplos que subsanen las deficiencias de competencia del usuario.

\subsubsection{Módulo dominio}

Contiene una representación computacional de un experto en el área de Números Naturales. Este conocimiento permite al STI comparar las acciones del estudiante y las selecciones con las de un experto a fin de evaluar lo que el usuario conoce y lo que necesita aprender. Una variedad de técnicas de Inteligencia Artificial se utilizan para la captura, de cómo un problema puede ser resuelto. Por ejemplo, se capta el conocimiento del estudiante a base de reglas que permite que el STI genere problemas sobre la marcha. Además permite que el desarrollador del curso pueda crear plantillas, que especifican una secuencia permitida de acciones correctas. Este método evita la codificación de la capacidad de resolver todos los problemas posibles. En cambio, sólo requiere la capacidad de especificar cómo el alumno deberá responder en un escenario.

\subsubsection{Módulo estudiante}

Evalúa el desempeño de cada alumno para determinar sus conocimientos, habilidades perceptivas y habilidades de razonamiento. Al mantener y hacer referencia a un módulo con el detalle de las fortalezas y las debilidades de los usuarios, el STI puede ofrecer muy específicamente, la instrucción pertinente. 


\subsubsection{Estilos de aprendizaje}

Para realizar el manejo de los estilos de aprendizaje se utiliza el test de Felder-Silverman [13], el cual consta de 44 preguntas. El estilo de aprendizaje del estudiante se almacena en su perfil académico y con esta información el STI selecciona las lecciones que presenta al estudiante de tal manera que el estudiante pueda aprender de acuerdo a su estilo de aprendizaje, en este caso solo se maneja la dimensión relativa al tipo de estímulos preferenciales que corresponde a estilo verbal y visual.

\subsubsection{Gestión de usuarios}

Es el componente que accede la capa de servicios para que la persistencia de la información sea totalmente encapsulada y el acceso se vea comprensible y simple.

\subsubsection{Módulo neurodifusor}

Contiene a las clases necesarias para la lógica difusa y la conexión hacia el componente afectivo para proporcionarle la información necesaria para que el tutor tome las decisiones correspondientes.

\section{Lógica difusa}

El STI utiliza la teoría de conjuntos difusos y reglas difusas para calibrar lo vago o ambiguo en las 4 variables difusas que maneja, como son tiempo, emoción, nivel en el ejercicio como variables de entrada y la cuarta es la variable de salida, que se llama nivel de dificultad, la cual será tomada en cuenta para el próximo ejercicio que se le presentará al estudiante.

Un conjunto difuso puede ser definido simplemente como un conjunto con límites difusos.[22]

Visto de otra manera, en la teoría difusa, un conjunto difuso $A$ de universo $X$ esta definido por la función $\mu_{A}(x)$ llamada función de membresía del conjunto $A$.

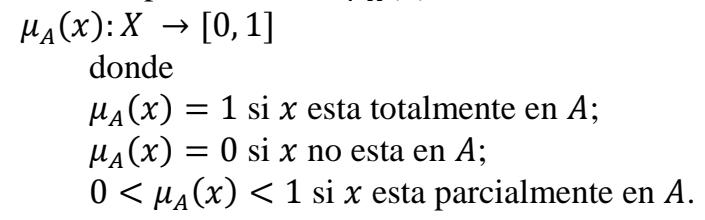

Una regla difusa puede ser definida como un enunciado condicional con variables lingüísticas y valores lingüísticos determinados por los conjuntos difusos en el universo del discurso.

Algunas reglas difusas que se manejan en el sistema son las siguientes:

1. If (nivelEnEjercicio is bajo) and (tiempoResolviendo is lento) and (estadoAfectivo is enganchado) then (dificultadEjercicio is baja)

2. If (nivelEnEjercicio is regular) and (tiempoResolviendo is rapido) and (estadoAfectivo is distraido) then (dificultadEjercicio is alta) 
3. If (nivelEnEjercicio is regular) and (tiempoResolviendo is lento) and (estadoAfectivo is neutral) then (dificultadEjercicio is baja)

4. If (nivelEnEjercicio is regular) and (tiempoResolviendo is regular) and (estadoAfectivo is enganchado) then (dificultadEjercicio is alta)

5. If (nivelEnEjercicio is bueno) and (tiempoResolviendo is rapido) and (estadoAfectivo is enganchado) then (dificultadEjercicio is alta)

\subsection{Capa servicios técnicos}

Contiene todos los servicios de bajo nivel, como lo son base de datos y comunicación entre sistemas y componentes de detección de emociones. Esta capa cuenta con tres componentes que se describen a continuación.

Persistencia: Maneja todo lo relacionado con base de datos, debido a que en ninguna otra parte del sistema tutor existen accesos para guardar la información. Este componente provee las interfaces necesarias para el guardado y al ser utilizada una tecnología ORM este puede guardar para distintos proveedores.

Módulo Afectivo: Se encarga de procesar las imágenes y devolver la emoción que se detectó hacia la capa superior, proponiendo así una API de fácil acceso.

Comunicaciones: Se utiliza para la interacción con otros sistemas, como lo es el log in para Facebook.

\section{$5 \quad$ Pruebas y evaluación del STI}

El STI aún no ha sido probado con estudiantes ya que hasta este momento se está finalizando la integración de los módulos que lo conforman.

Alternativamente, se decidió realizar una evaluación comparativa con otros sistemas tutores que tienen una finalidad similar y el STI para Tercer Grado de Matemáticas. Ver Tabla 2. Evaluación y comparación de características propias de los STI's con el Sistema Tutor Inteligente Afectivo y Configurable para el aprendizaje de los números naturales en 3er. Grado, de acuerdo a la escala de Likert. En esta se listan diversas características de los sistemas tutores inteligentes y se propone una evaluación usando una escala de Likert con valores de 5 a 1 representando Totalmente de acuerdo hasta totalmente en desacuerdo respectivamente.

Tabla 2. Evaluación y comparación de características propias de los STI’s con el Sistema Tutor Inteligente Afectivo y Configurable para el aprendizaje de los números naturales en 3er. Grado, de acuerdo a la escala de Likert.

\begin{tabular}{lllll}
\hline $\begin{array}{l}\text { Característica del } \\
\text { Tutor Inteligente }\end{array}$ & Descripción de la característica & CTAT & $\begin{array}{l}\text { Animal } \\
\text { Watch }\end{array}$ & $\begin{array}{l}\text { Active } \\
\text { Math }\end{array}$ \\
\hline
\end{tabular}




\begin{tabular}{|c|c|c|c|c|c|}
\hline Generatividad & $\begin{array}{c}\text { Capacidad de generar problemas } \\
\text { apropiados, pistas y ayuda necesaria, adaptados } \\
\text { a las necesidades de aprendizaje del estudiante. } \\
\text { Capacidad de representar y razonar acerca }\end{array}$ & 3 & 5 & 4 & 5 \\
\hline $\begin{array}{l}\text { Modelo del } \\
\text { estudiante }\end{array}$ & $\begin{array}{l}\text { del conocimiento del estudiante y las } \\
\text { necesidades de aprendizaje en respuesta a la } \\
\text { proporción de instrucciones. }\end{array}$ & 3 & 5 & 4 & 5 \\
\hline Modelo del experto & $\begin{array}{l}\text { Representación y ruta de razonamiento } \\
\text { acerca del rendimiento dentro del dominio y la } \\
\text { capacidad implícita de respuesta por instrucción } \\
\text { proporcionada. }\end{array}$ & 5 & 5 & 3 & 5 \\
\hline Iniciativa Mixta & $\begin{array}{l}\text { Capacidad de iniciar la interacción con el } \\
\text { estudiante, así como la interpretación y } \\
\text { respuesta de manera útil a interacciones } \\
\text { iniciadas por los estudiantes. }\end{array}$ & 5 & 3 & 4 & 4 \\
\hline $\begin{array}{l}\text { Aprendizaje } \\
\text { interactivo }\end{array}$ & $\begin{array}{l}\text { Actividades de aprendizaje que requieren } \\
\text { compromiso y autenticidad contextualizados en } \\
\text { el dominio apropiado. }\end{array}$ & 5 & 4 & 4 & 5 \\
\hline $\begin{array}{l}\text { Modelo } \\
\text { instruccional }\end{array}$ & $\begin{array}{l}\text { Capacidad de cambiar el modo de } \\
\text { enseñanza basado en inferencias sobre el } \\
\text { aprendizaje del alumno. }\end{array}$ & 4 & 4 & 4 & 3 \\
\hline Auto-mejora & $\begin{array}{l}\text { Capacidad del sistema para monitorear, } \\
\text { evaluar y mejorar su propia enseñanza basado } \\
\text { en su experiencia con otros alumnos. }\end{array}$ & 3 & 3 & 3 & 3 \\
\hline
\end{tabular}

\section{Conclusiones}

Durante el desarrollo se buscó implementar todas las características que requiere un buen STI, estas fueron: reconocimiento afectivo, consideración de estilos de aprendizaje, reacción inteligente hacia las necesidades cognitivas del estudiante y configuración por parte de los expertos.

Se evaluaron las características propuestas con las características implementadas y se concluyó que el potencial de un STI que maneje lo último en tecnologías de configuración, tiene mayor capacidad de influencia en el sector educativo puesto que se puede adaptar a entornos tan variados como son los que hay actualmente en México.

Otra de las aportaciones valiosas en el proyecto fue la implementación del acceso a través de las redes sociales, las cuales son utilizadas día con día por un gran número de estudiantes, lo cual abre un gran nicho para la participación del STI.

En trabajos futuros se pretende aplicar un mayor número de estilos de aprendizaje a las lecciones, agregar otras maneras de reconocer el estado cognitivo del estudiante, para que no solo expertos en lógica difusa puedan modificarlo. Además de mejorar el agente pedagógico, para que sea capaz de ser configurado para responder a las entradas del estudiante por voz. Actualmente se esta trabajando en el diseño de un experimento en conjunto con una escuela primaria de la localidad, para probar el funcionamiento y verificar la eficacia de los métodos aplicados en el STI. 


\section{Referencias}

[1] Aleven, V., Koedinger, K.: An effective metacognitive strategy: learning by doing and explaining with a computer-based cognitive tutor. Cognitive Science, 26(2), 147-179, (2002).

[2] Anderson, R., Boyle, C. F., Corbett, A. T., \& Lewis, M. W.: Cognitive modeling and intelligent tutoring. Artificial Intelligence, 42, 17-49. Doi: 10.1016/0004-3702(90)90093-F, (1990).

[3] Carbonell, J. R.: AI in CAI: An artificial intelligence approach to computer-aided-instruction. IEEE Transactions on Man-Machine System. MMS, 11(4), 190-202, (1970).

[4] Clancey, W. J.: Transfer of rule-based expertise through a tutorial dialogue. Ph.D. thesis, Department of Computer Science, Stanford University, (1979).

[5] Woolf, B. P., Building intelligent interactive tutors. Morgan Kaufmann, (2009).

[6] Ekman, P. Are there basic emotions? Psychological Review, 99, 550-553, (1992).

[7] Mandler, G. Emotion. In B. M. Bly \& D. E. Rumelhart (Eds.), Cognitive science. Handbook of perception and cognition 2nd ed. (pp. 367-384). San Diego, CA: Academic Press, (1999).

[8] Arroyo, I., Woolf, B., Cooper, D., Burleson, W., Muldner, K., Christopherson, R.: Emotions sensors go to school. In: Proceedings of the 14th international conference on artificial intelligence in education (AIED 2009), Brighton, UK, pp. 17-24. IOS press, Amsterdam,( 2009).

[9] Zatarain-Cabada, R., Barrón-Estrada, M.L., Beltrán, J.A., Cibrian, F.L., Reyes-García, C., Hernández, Y.: Fermat: merging affective tutoring systems with learning social networks. In: Proceedings of the 12th IEEE international conference on advanced learning technologies, Rome, Italy, 337-339. IEEE Computer Society, (2012).

[10] Doignon, J. -P. and Falmagne, J. C.: Knowledge Spaces. Springer-Verlag, (1999).

[11] Günel, K.: Intelligent Tutoring Systems: conceptual map modeling. Lambert Academic Publishing, (2010).

[12] Felder, R. M., \& Solomon, B. A.: Index of Learning Styles Questionnaire, http://www.engr.ncsu.edu/learningstyles/ ilsweb.html, Retrieved Octuber 4, (2013).

[13] Felder, R. M., \& Silverman, L.K.: Learning and teaching styles in engineering education, Engineering Education, pp. 674-681, vol. 78, (1988).

[14] Woolf, BP. Building intelligent interactive tutors: Student-centered strategies for revolutionizing elearning, (2010).

[15] Beal, CR, Arroyo, I., Cohen, PR, Woolf, BP, \& Beal, CR.: Evaluation of AnimalWatch: An intelligent tutoring system for arithmetic and fractions. Journal of Interactive Online Learning , 9 (1), 64-77, (2010).

[16] Beal, C. R., Adams, N. M., \& Cohen, P. R. Reading proficiency and mathematics problem solving by high school English Language Learners. Urban Education, 45(1), 58-74, (2010).

[17] Fournier-Viger, P., Nkambou, R., Nguifo, E. M., \& Mayers, A.: ITS in ill-defined domains: toward hybrid approaches. In Intelligent Tutoring Systems, Springer Berlin Heidelberg, pp. 318-320, (2010).

[18] D’Mello, S. K. \& Graesser, A. C.: AutoTutor and affective AutoTutor: Learning by talking with cognitively and emotionally intelligent computers that talk back. ACM Transactions on Interactive Intelligent Systems, 2(4), 23:2-23:39, (2012).

[19] Olney, A. M., D’Mello, S., Person, N., Cade, W., Hays, P., Williams, C., ... \& Graesser, A. (2012, January). Guru: A computer tutor that models expert human tutors. In Intelligent Tutoring Systems, Springer Berlin Heidelberg, (pp. 256-261), (2010).

[20] Aleven, V., McLaren, B. M., Sewall, J., \& Koedinger, K. R.: The cognitive tutor authoring tools (CTAT): Preliminary evaluation of efficiency gains. In Intelligent Tutoring Systems, Springer Berlin Heidelberg, (pp. 61-70), (2006).

[21] Melis, E., \& Siekmann, J.: Activemath: An intelligent tutoring system for mathematics. In Artificial Intelligence and Soft Computing-ICAISC 2004, Springer Berlin Heidelberg, pp. 91-101, (2004).

[22] Negnevitsky, M.: Artificial Intelligence a guide to intelligent systems. 2a . Pearson Education, (2005).

[23] Secretaria de Educación Pública. Matemáticas Tercer Grado. México : Chanti Editores, (2012). 